Classification

Physics Abstracts

$81.20 \mathrm{~S}-61.50 \mathrm{C}-36.20$

\title{
Crystallization of quenched polyethylene. Part III : Mixtures of fractions
}

\author{
J. Rault \\ Laboratoire de Physique des Solides, Bât. 510, Université Paris-Sud, 91405 Orsay, France \\ and E. Robelin \\ Institut Textile de France, 92100 Boulogne, France
}

(Reçu le 17 septembre 1981, révisé le 2 mars 1982, accepté le 3 juin 1982)

\begin{abstract}
Résumé. - La cristallisation des mélanges de polyéthylène fractionnés par trempe du fondu a été étudiée par la diffusion des Rayons $\mathrm{X}$ aux petits et grands angles et par analyse thermique différentielle. Les lois de variation des longues périodes avec les masses moléculaires en nombre et en poids sont données. On montre que les longues périodes sont données par la moyenne en poids de la distance bout à bout $r_{\mathrm{w}}$ des pelotes dans le fondu juste avant la cristallisation. Dans les mélanges les chaînes longues de poids moléculaire supérieur à $M^{*}=10^{5}$ peuvent être décomposées en une série de chaînes indépendantes de masse critique $M^{*}$. La cristallinité des mélanges mesurée par RX et par ATD suit les variations de la cristallinité linéaire $l_{\mathrm{c}} / L$ où $l_{\mathrm{c}}$ est l'épaisseur des lamelles cristallines, indépendante de la masse moléculaire. Ces corrélations entre l'état solide et fondu montrent que les caractéristiques de l'état semi-cristallin ne sont pas contrôlées par les phénomènes de nucléation secondaire, mais par les caractéristiques dimensionnelles des pelotes juste avant cristallisation. Ces résultats montrent que les processus de cristallisation des chaînes courtes et longues sont différents, ainsi on expliquerait les différences observées dans les cinétiques de cristallisation, et dans la morphologie de l'état semi-cristallin lorsque la masse moléculaire est changée.
\end{abstract}

\begin{abstract}
The crystallization of mixtures of P.E. fractions quenched from the melt has been studied by SAXS, WAXS and DTA. The laws of variation of the long period with the weight and number average molecular weights are given. It is shown that the long period, $L$, of the semi-crystalline state is given by the weight average of the end-to-end distance $r_{\mathrm{w}}$ of the coils in the liquid state before quenching. For mixtures containing high molecular weight $M>M^{*}=10^{5}$, the long chains behave like a series of segments of chains of molecular weight $M^{*}$ which crystallize independently. The crystallinity measured by WAXS and DTA varies with the molecular weight and its distribution as the linear crystallinity $l_{\mathrm{c}} / L \sim l_{\mathrm{c}} / r_{\mathrm{w}}$ where $l_{\mathrm{c}}$ is the crystalline lamella thickness and is independent of the molecular weight. The correlations emphasized in this paper between the solid and melt states of P.E. support the contention that the structure of lamellar semi-crystalline P.E. is not controlled by the phenomenon of secondary nucleation, but by the dimensions and the rigidity of the coils just before crystallization. The results reported in this paper show that the process of crystallization of short and long chains are different; that would explain the differences observed in studies of the kinetics of crystallization, and in the morphology of the semicrystalline state when the molecular weight is increased.
\end{abstract}

1. Introduction. - We have reported recently $[1,2,3]$ the correlations existing between the solid state and the melt of polyolefines. In quenched polyethylene fractions of molecular weight $M<M^{*}$, the long period $L$ varies as the end-to-end distance $r_{0}$ of the coils in the liquid state, and is of the same order of magnitude. Above the critical molecular weight $M^{*}$, the long period does not vary. The laws of variations of $L$ with molecular weight [2] are given by the relations :

$$
L \sim r_{0}=\sqrt{M} \quad 10^{4}<M<M^{*}=10^{5}
$$

$$
L \sim r^{*}=\sqrt{M^{*}} \quad M \geqslant M^{*}=10^{5}
$$

$L$ and $r$ are given in angström. For very low molecular weight material, $M<2000$, the long periods are given by the length of the chains [ $4 a, 37]$, very short chains crystallize like parafines. The domain $10^{4}<M<10^{5}$ will be called the domain of low molecular weight materials, in that domain the exact law giving the long periods as a function of the molecular weight is [2] :

$$
L=130 \AA+0.54 \sqrt{M} .
$$


In the melt, when equilibrium is reached, the polymer chains are in the ideal state and the end-to-end distance of the chains is a function of the melt temperature $T_{\mathrm{i}}$. Therefore, these relations imply that in quenched fractions the long period is a function of the melt temperature, just before crystallization. This effect has been analysed in Parts I [1] and II [2]. In Part II, the solid coefficient $\alpha=\frac{1}{L} \frac{\partial L}{\partial T_{\mathrm{i}}}$ has been compared to the Flory temperature coefficient of the melt $\beta=\frac{2}{r_{0}} \frac{\partial r_{0}}{\partial T_{\mathrm{i}}}$. The experimental relationship $\beta=2 \alpha$ between the two coefficients characteristic of the solid and liquid phases verifies relation (1).

In quenched polydispersed commercial materials, in general the long period is believed to be independent of the molecular weight and its distribution. In fact, this is not true, small differences in the long period are observed in materials presenting small differences in the molecular weight distribution [5] and important differences when the molecular weight distributions are very different [2].

Kinetic theories [4a] do not explain this paramount property; neither do they explain or predict the amount of amorphous phase and the crystallinity in semi-crystalline materials. In polydispersed materials such as commercial products, the relationship between long period and molecular weight (number average $M_{\mathrm{N}}$ and weight average $M_{\mathrm{w}}$ ) is not obvious. But $L$ is still an increasing function of $M_{\mathrm{N}}$ and $M_{\mathrm{w}}$. The correlation between the solid state and the liquid state of several polymers of different molecular weight distributions have been studied in Part I. For polydispersed P.E., the relationship between the solid and liquid coefficients $\alpha$ and $\beta$ is somewhat different from that obtained for P.E. fractions. However, these two parameters are of the same sign and of the same order of magnitude.

In order to understand the crystallization of highly polydispersed polymers and for comparison with the crystallization of fractionated polymers, it is necessary to study mixtures of fractions having an intermediate degree of polydispersity. This is the aim of this paper.

By mixing fractions, the molecular weights $M_{\mathrm{N}}$ and $M_{\mathrm{w}}$ and therefore the polydispersity $I=M_{\mathrm{w}} / M_{\mathrm{N}}$ were varied quasi-continuously. The average long period, the fluctuations of the long period and the crystallinity of these mixtures are correlated to the molecular weight distribution and thereafter to the end-to-end distance distribution of the liquid polymer chains just before crystallization. The observation of similar variations of the long period and the weight average end-to-end distance permits an explanation of the dependence of the crystallinity on the molecular weight and the polydispersity.

2. Experimental procedure. - The number average molecular weight $M_{\mathrm{N}}$ and the polydispersity $I$ of the fractions used are given in the following table :

\begin{tabular}{|c|r|r|r|r|r|}
\hline Sample & $10 \mathrm{~K}$ & \multicolumn{1}{|c|}{$30 \mathrm{~K}$} & $60 \mathrm{~K}$ & $100 \mathrm{~K}$ & \multicolumn{1}{c|}{$260 \mathrm{~K}$} \\
\hline$M_{\mathrm{N}}$ & 9950 & 30700 & 59000 & 112000 & 246000 \\
\hline$I$ & 1.1 & 1.1 & 1.09 & 1.10 & 1.15 \\
\hline
\end{tabular}

The samples used were made by combining various amounts of 2 of the fractions. The fractions were dissolved in xylene at $120^{\circ} \mathrm{C}$ for $20 \mathrm{~min}$. with stirring and the solvent was then evaporated in vacuum. The mixtures were then held molten at $150^{\circ} \mathrm{C}$ in films of $1 \mathrm{~mm}$ thickness for $2 \mathrm{~h}$ and quenched in liquid nitrogen. The cooling rate is about $20^{\circ} \mathrm{C} . \mathrm{s}^{-1}$.

Some samples were quenched in water at $20^{\circ} \mathrm{C}$ and at $0^{\circ} \mathrm{C}$. They present the same long period as those quenched in liquid nitrogen. This shows that for high cooling rate the long period is independent of the cooling rate [2]. It is well known that for slow cooling rate $L$ is not independent of the cooling rate $[4,55]$. The interest of such quenching experiments is to obtain reproducible values of $L$ correlated to the dimension of the coils just before crystallization as observed in reference [2].

The small angle X-ray scattering of quenched mixtures were recorded at LURE with the synchrotron wavelength $\lambda=1.9 \AA$ and a sample-counter distance of $1 \mathrm{~m}$. This experimental set-up allows one to determine with accuracy the location of the maximum of the Bragg peak and the relative width $\Delta \theta / \theta$ of the peak. The precision on the measurements of the Bragg long period is $\Delta L / L \sim 10^{-2}$, and the precision on $\Delta \theta / \theta$ is about 0.03 . This precision can be observed in figure 1 of reference [2].

As in Parts I and II, we are not interested in exact values of the long period but rather in variations with the different molecular weights $M_{\mathrm{N}}$ and $M_{\mathrm{w}}$. The evaluation of the long period is therefore restricted to a measurement of the maximum of the Bragg peak; background corrections were made only for high molecular weight materials presenting Bragg spacing greater than $300 \AA$. The crystallinity was determined by two methods : wide angle $\mathrm{X}$-ray scattering and differential thermal analysis. The index of crystallinity was determined by WAXS according to the arbitrary procedure given in reference [6].

The reproducibility of the measurement is about $1 \%$, but the difference between this index of crystallinity and the crystallinity determined by DTA is of the order of $5 \%$.

3. Results. - 3.1 LONG PERIODS. - Because of the existence of two distinct regimes in fractionated P.E. crystallization described by relations (1) and (2), we will distinguish three types of binary mixtures, according to the values of the molecular weights $M_{x}$ and $M_{y}$ of the low and high molecular weight fractions compared to the critical value $M^{*}=10^{5}$. Figures 1a-e give the Bragg long periods and the 

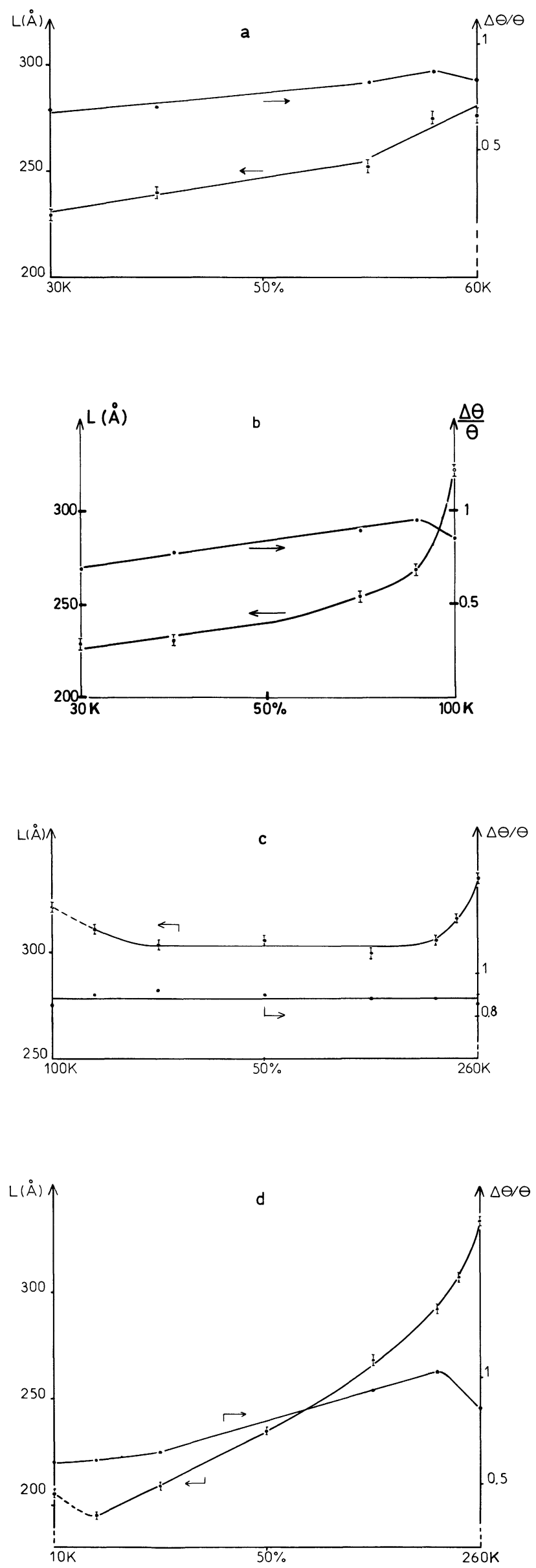

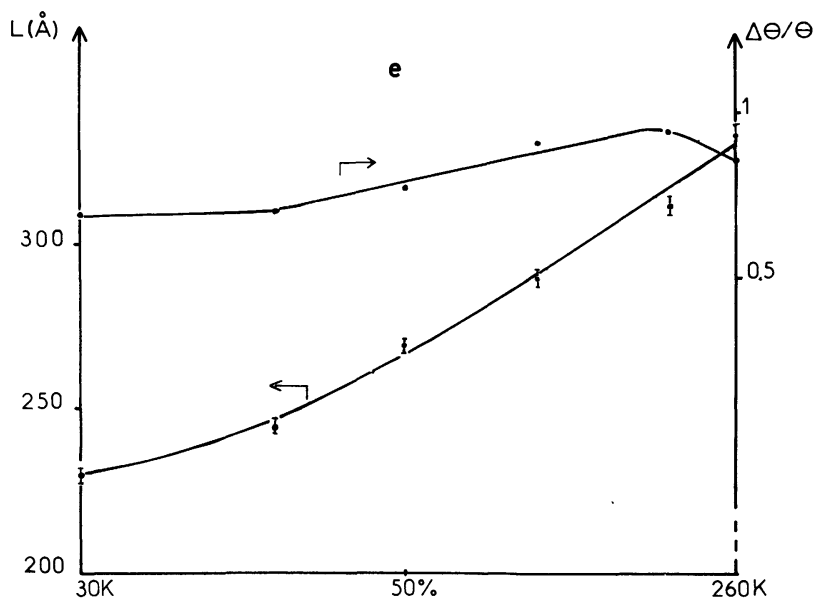

Fig. 1. - Long period $L$ and half width $\Delta \theta / \theta$ of the SAXS intensity peak of different mixtures as a function of the mass concentration : a) $30 \mathrm{~K}-60 \mathrm{~K}$; b) $30 \mathrm{~K}-100 \mathrm{~K}$; c) $100 \mathrm{~K}$ $260 \mathrm{~K}$; d) $10 \mathrm{~K}-260 \mathrm{~K}$; e) $30 \mathrm{~K}-260 \mathrm{~K}$.

relative width of the Bragg peak of mixtures as a function of the mass concentration $M_{y}$ of long chains. Figures 2 and 3 give the dependence of the long period with the square root of the number and weight average molecular weights. The line AB represents the long period of the pure fractions of molecular weight smaller than $M^{*}$ (see Fig. 3 of Ref. [2]).

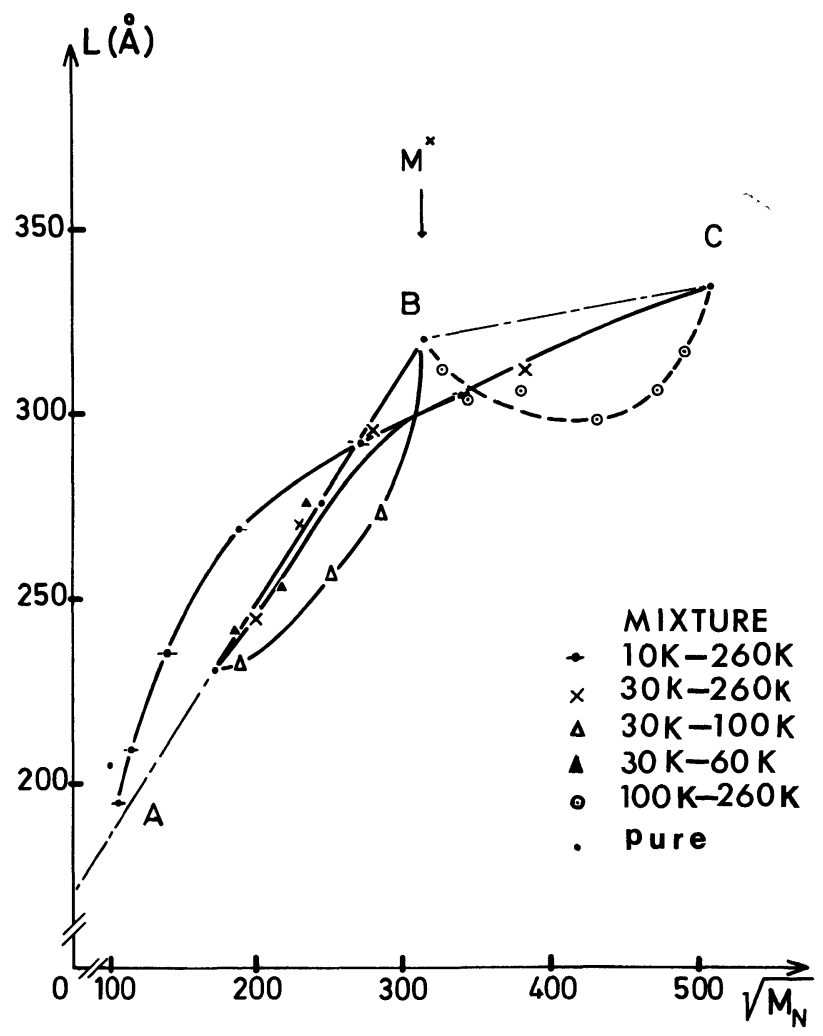

Fig. 2. - Long period of the different mixtures of figure 1 expressed as functions of $\sqrt{M_{\mathrm{N}}}$. The long periods of pure fractions obey to relations (1) and (2) and define the straight lines $\mathrm{AB}$ and $\mathrm{CD}$. 


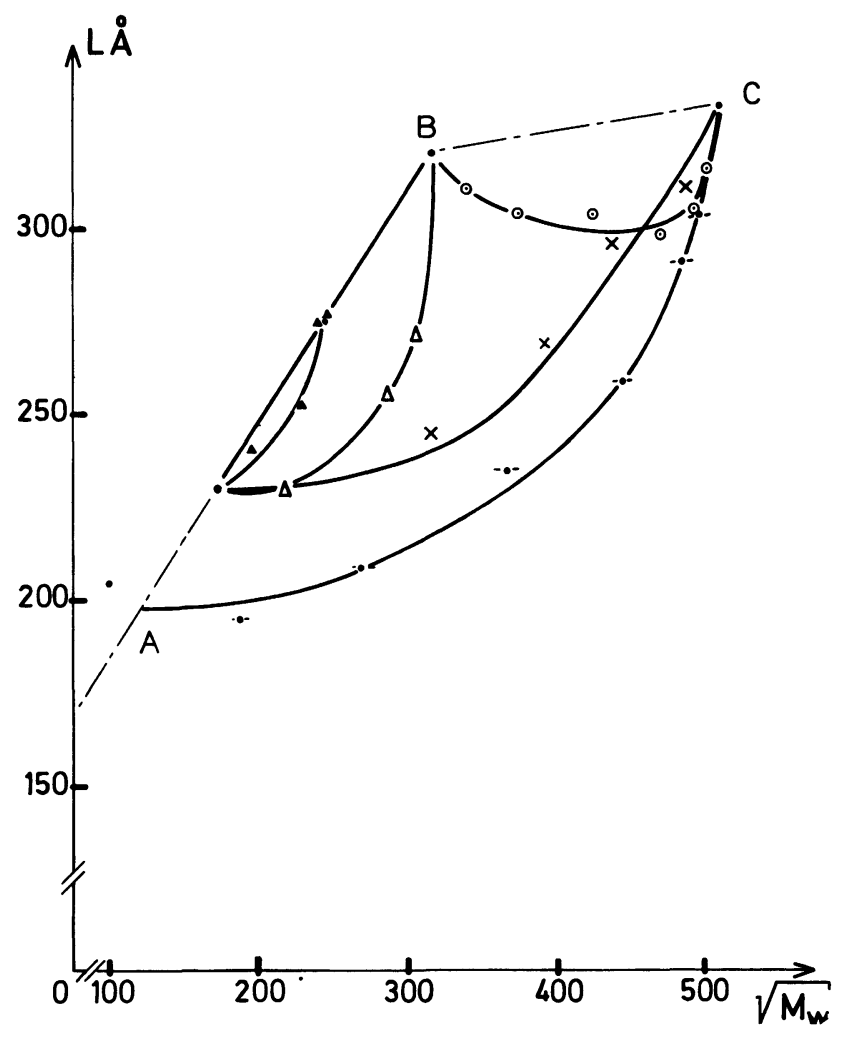

Fig. 3. - Long period of the mixtures expressed as function of $\sqrt{M}_{\mathrm{w}}$. The linear curves $\mathrm{AB}$ and CD correspond to the values of fractionated samples, see figure 3 of reference [2].

a) Mixtures of fractions of low molecular weight $M_{x}, M_{y}<M^{*}$. - The comparison between the mixtures $30 \mathrm{~K}-60 \mathrm{~K}$ and $30 \mathrm{~K}-100 \mathrm{~K}$ shows the importance of the polydispersity. The larger the difference $M_{y}-M_{x}$, the larger the difference between the laws of variations $L\left(M_{\mathrm{N}}\right)$ and $L\left(M_{\mathrm{w}}\right)$, and the larger the difference between the actual dependence of $L$ on $\sqrt{M}_{\mathrm{N}}$ and $\sqrt{M}_{\mathrm{w}}$ and a linear dependence. Because of the small difference between $M_{\mathrm{N}}$ and $M_{\mathrm{w}}$ for the mixture $30 \mathrm{~K}-60 \mathrm{~K}$, the long period follows the curve $\mathrm{AB}$ of fractionated samples given by relation (1) and a linear dependence of $L$ with $\sqrt{M}_{\mathrm{N}}$ or $\sqrt{M}_{\mathrm{w}}$ is equally valid.

b) Mixtures of high molecular fractions $M_{x}$, $M_{y}>M^{*}$. - Individual fractions with $M>M^{*}$ show no dependence of $L$ with the molecular weight. The long periods are not given by the end-to-end distance $r_{0}$ of the coils.

In the mixtures $100 \mathrm{~K}-260 \mathrm{~K}$, the same trend is observed. It should be noted that, for high molecular weight, the lamellae are highly curved and that the spherulitic organization is highly deteriorated [28]. The distribution of the long period can be very different from that of low molecular weight materials. The interpretation of the small variation observed is therefore not a simple task. It should be noted here again that there is not much difference between the curves $L\left(M_{\mathrm{N}}\right)$ and $L\left(M_{\mathrm{w}}\right)$ because of the small value of the polydispersity (which reaches 1.25 for $50 \%$ mixture of $M_{x}=100 \mathrm{~K}$ and $M_{y}=260 \mathrm{~K}$ ).

c) Mixtures of low and high molecular weight $M_{x}<M^{*}<M_{y^{*}}$ - The mixtures $10 \mathrm{~K}-260 \mathrm{~K}$ and $30 \mathrm{~K}-260 \mathrm{~K}$ have high polydispersity, for example the mixtures at $50 \%$ have respectively polydispersity of 7 and 2.7 to be compared to values of 4 to 10 for many commercial products. Here the prominent feature is the difference between the curves $L\left(M_{\mathrm{N}}\right)$ and $L\left(M_{\mathrm{w}}\right)$ and the large deviation from the linear curves for the fractionated samples as given by relations (1) and (2). The larger the polydispersity, the larger the deviation.

3. 2 Relative WidTh $\Delta \theta / \theta$ OF THE BRAGG PEAK. The relative width for fractionated samples is reported in figure 4 as a function of the square root of the molecular weight. Taking into account the accuracy of $\Delta \theta / \theta$, the break in the curve corresponds closely to the mass $M^{*}$ defined in figure 2 as separating the two domains of low and high molecular weight.

For the high molecular weight $100 \mathrm{~K}-260 \mathrm{~K}$, the relative width is nearly constant (Fig. 1e). This fact and the relative constancy of the long period indicate that the structure of the semi-crystalline state does not depend on the molecular weight when all the chains have a molecular weight higher than $M^{*}$. This fact is to be related to the independence of the growth rate with the molecular weight for high molecular weight materials [7] and to the independence of the density and surface energy with $M_{N}$ for materials having a number molecular weight higher than a critical value of the order of $M^{*}$ [8].

For low molecular weight mixtures the relative width is an increasing function of the coil concentration $c_{y}$ of the high molecular weight fraction $\left[c_{y}=\frac{n_{y}}{n_{x}+n_{y}}, n_{x}\right.$ and $n_{y}$ being respectively the numbers of chains of mass $M_{x}$ and $M_{y}$ ]. A maximum is noted in any case for a high value of $c_{y} \sim 90 \%$. This

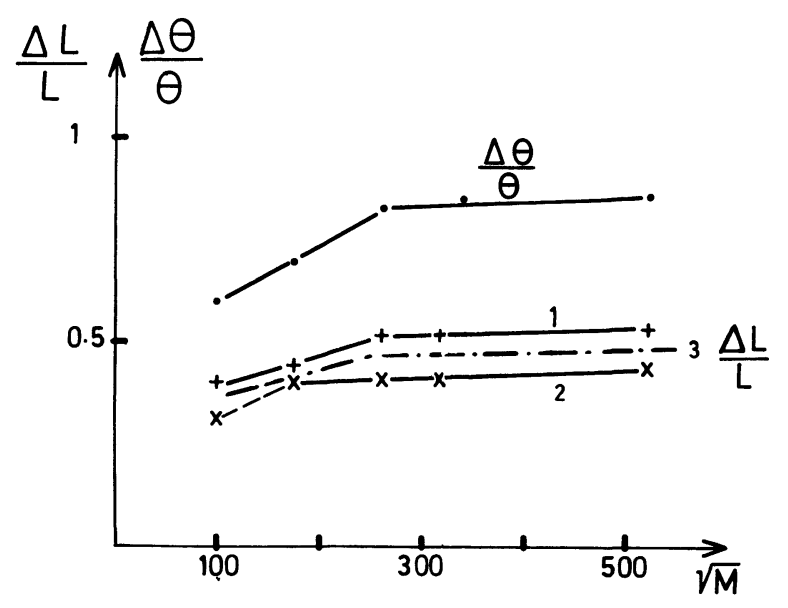

Fig. 4. - Relative width $\Delta \theta / \theta$ of the SAXS intensity peak, and dispersity of the long period deduced from Tsvankin (2) and Slutsker $(1,3)$ analysis as a function of the square root of the molecular weight. 
effect shows that the polydispersity decreases somewhat the regularity of the lamella structure.

The value of the relative width $\Delta \theta / \theta \simeq 0.8$ observed here for high molecular weight fractions and mixtures is of the same order of magnitude as that for polydispersed commercial P.E. and for other semi-crystalline polymeric materials [9]. This parameter, characteristic of the solid state, will be related below to a parameter characteristic of the liquid chain just before crystallization.

4. Discussion. - 4.1 INTERPRETATION OF THE VARIATION OF THE LONG PERIOD. - The paramount question is to find if relation (1) holds for mixtures of fractionated P.E. when $M$ is replaced by an average molecular weight, or in other words if there is a linear relationship between the measured long period and an average value of the end-to-end distance of the coils in the melt. As X-ray scattering is sensitive to the mass of scattering objects, it is expected that the long period would be governed by the weight average of the endto-end distance rather than by the number average.

Consider a distribution of molecular weight $f\left(M_{x}\right)$ and $\omega_{\mathrm{i}}$ the mass concentration of species $M_{\mathrm{i}}$. For polyethylene the end-to-end distance [32] of species $M_{\mathrm{i}}$ is, in angströms, $r\left(M_{\mathrm{i}}\right)=0.95 \sqrt{M_{\mathrm{i}}} \sim M_{\mathrm{i}}^{1 / \overline{2}}$ [32].

The weight average of the end-to-end distance is :

$$
r_{\mathrm{w}}=\sum_{\mathrm{i}} \omega_{\mathrm{i}} M_{\mathrm{i}}^{1 / 2}
$$

$r_{\mathrm{w}}$ is equal to the moment $+1 / 2$ of the molecular weight distribution.

The moments $(-1)$ and $(+1)$ give the number and weight average of the molecular weight according to the following expressions :

$$
\begin{aligned}
& M_{\mathrm{N}}^{-1}=\sum_{\mathrm{i}} \omega_{\mathrm{i}} M_{\mathrm{i}}^{-1} \\
& M_{\mathrm{w}}=\sum_{\mathrm{i}} \omega_{\mathrm{i}} M_{\mathrm{i}} .
\end{aligned}
$$

The number average of the end-to-end distance $r_{\mathrm{N}}$ is :

$$
r_{\mathrm{N}}=\sum_{\mathrm{i}} c_{\mathrm{i}} M_{\mathrm{i}}^{1 / 2}
$$

where $c_{\mathrm{i}}$ is the number concentration of coils of species $M_{\text {i }}$

$$
c_{\mathrm{i}}=\omega_{\mathrm{i}} / M_{\mathrm{i}} \times\left[\Sigma \omega_{\mathrm{i}} / \boldsymbol{M}_{\mathrm{i}}\right]^{-1}
$$

The polydispersity $I$ of the mixtures will be defined as the ratio of the two molecular weights $M_{\mathrm{N}}$ or $M_{\mathrm{w}}$ of the fractions, the polydispersity of the fraction will not be taken into account. This simplification for calculating $I$ will not change our conclusions.

The following table gives the maximum polydispersity of the mixtures (i.e. having a mass concentration of $50 \%$ of each fraction).

\begin{tabular}{|c|c|c|c|c|}
\hline $30 \mathrm{~K}-60 \mathrm{~K}$ & $100 \mathrm{~K}-260 \mathrm{~K}$ & $30 \mathrm{~K}-100 \mathrm{~K}$ & $30 \mathrm{~K}-260 \mathrm{~K}$ & $10 \mathrm{~K}-260 \mathrm{~K}$ \\
\hline 1.12 & 1.25 & 1.4 & 2.7 & 7 \\
\hline
\end{tabular}

a) Mixtures of low polydispersity. - The first three mixtures have a maximum of polydispersity which is comparable to the polydispersity of the individual fractions, i.e. 1.1.

For these mixtures there are only slight differences between $\sqrt{M}_{\mathrm{N}}, \sqrt{M}_{\mathrm{w}}, r_{\mathrm{N}}$ and $r_{\mathrm{w}}$, the variations of $L$ and $r_{\mathrm{N}}$ or $r_{\mathrm{w}}$ are quasilinear with $M_{\mathrm{N}}^{1 / 2}$ or $M_{\mathrm{w}}^{1 / 2}$ and equation (1) applies. For the mixtures of low molecular weight fractions with $M_{\mathrm{i}}<10^{5}$ the long periods are governed by the end-to-end distance of the coils in the liquid state before crystallization. Because of the comparable polydispersity of these mixtures with the polydispersity of each fraction, it is not possible from the experimental results to conclude if the paramount parameter controlling the long period is the number or the weight average of the end-to-end distance. This distinction will be drawn in the next paragraph in the case of mixtures of high polydispersity.

For mixtures of high molecular weight fractions, above the critical value $M^{*}=10^{5}$, the long period is not a linear function of $\sqrt{M}$. However, despite the observed variation between 300 and $350 \AA$, the long period will be considered as constant. The end-to-end distance of the coils are greater than the long period. Therefore, each polymer chain in our model passes through several adjacent crystalline lamellae. The number of tie molecules connecting adjacent crystalline layers increases with the molecular weight. The number of crystalline lamellae linked by a long chain $\left(M>M^{*}\right)$ is of the order of $r / L=\left(M / M^{*}\right)^{1 / 2}$. This property would explain the difference of morphology between low and high molecular weight fractions [28, 48, 49].

It is obvious that the mechanical properties depend on the number of tie molecules between adjacent crystallites; this has not been studied in P.E. fractions. However, it must be pointed out that a brittle-ductile transition appears in poly (Tetramethyl-p silphenylene) siloxane (T.M.P.S.) for high molecular weight material [7], and we expect that a similar transition should appear in P.E. It is certain as noted by Magill that the ease with which low molecular weight fraction materials fracture supports the contention that the intercrystallite links are very few in these materials [10].

The nearly constant long period for high molecular weight mixtures, and fractions indicates that the process of crystallization is independent of the length of chains; the chains can be separated into several segments of molecular weight $M^{*}$ which crystallize independently. Therefore, each portion with virtual ends having an end-to-end distance of $r^{*}=300 \AA$ gives rise by quenching to lamellae of thickness $L=r^{*}=300 \AA$. In figure 5 this process is schematized, each long chain in the melt state is replaced by a 

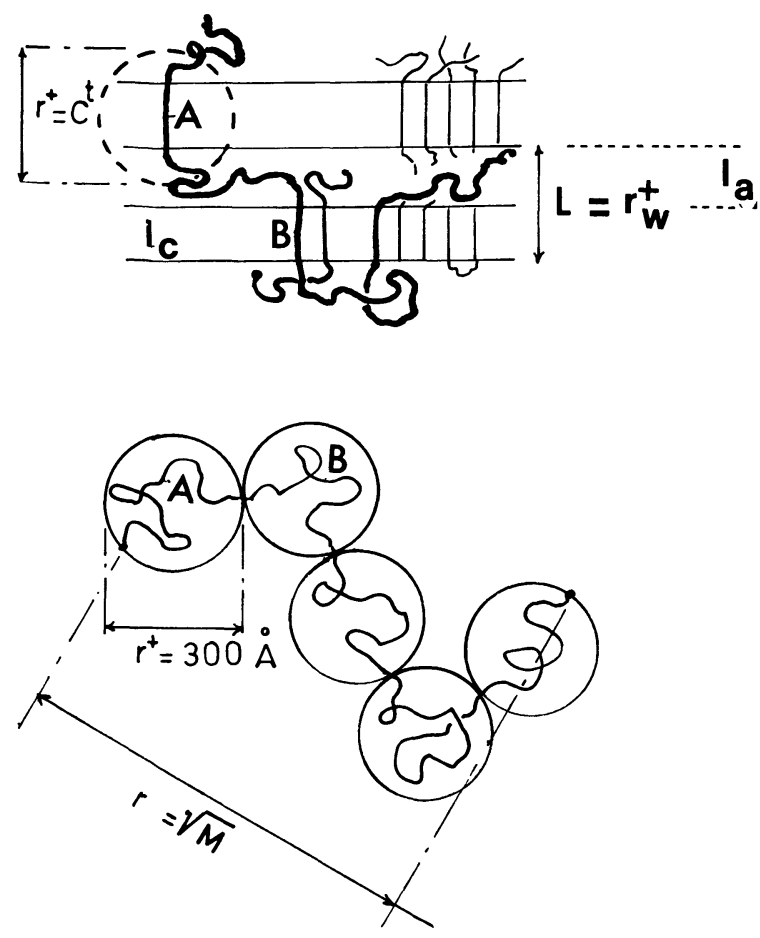

Fig. 5. - Decomposition of a long chain in smaller parts of molecular weight $M^{*}$. Each portion or blob of end-toend distance of the order of magnitude of $300 \AA$ crystallizes independently from each other. After crystallization, each blob is crossed by one crystalline lamellae of thickness $l_{\mathrm{c}}=150 \AA$. In the case of a mixture of small and long chains the long period is given by the weight average end-to-end distance $r_{\mathrm{w}}^{*}$. The chains having an end-to-end distance $r>r_{\mathrm{w}}^{*}$ must be considered as a tie molecule connecting several adjacent crystalline lamella.

series of adjacent blobs of radius $300 \AA$. After crystallization each blob is crossed by one crystalline lamellae. The dimension $r^{*}$ of the blob has no physical meaning in the melt; the segments of chain constituting the blobs interpenetrate in the melt and are in the ideal state. The reason for the appearance of this critical length $r^{*}$ is out of the scope of this paper; it is to be noted that kinetic reasons should be put forward. We suggest that, if the chain crystallizes at a certain place A, (nucleation or growth) it cannot crystallize at another place $\mathrm{B}$ if the distance $\mathrm{AB}$ is smaller than $r^{*}$. The distance $r^{*}$ can be considered as a perturbation distance around any crystallite that is to say the smallest distance from a crystallite where a second nucleus or crystallite can nucleate or grow (see paragraph 4.3).

It must be emphasized that this process of crystallization involving independent parts of the chains would explain why the rate of crystallization does not depend on the molecular weight $M$ for values of $M>M^{*}$. This property has been observed for P.E. fraction and T.M.P.S. [7]. For this latter polymer, the critical molecular weight above which the spherulitic growth rate is constant is of the order of $10^{5}$ (for a temperature of crystallization corresponding to the growth rate maximum). This value corresponds to the critical molecular weight above which the material becomes tough $(M=80000)$.

We think that P.E. fractions and T.M.P.S. fractions are similar in terms of their crystallization process. However, measurements of mechanical properties of P.E. fractions are highly desirable.

In conclusion, in high molecular weight P.E. quenched from the melt at a cooling rate of about $20^{\circ} \mathrm{C} / \mathrm{s}$, the crystallization process involves the movement of independent parts of the chains. Each long chain can be visualized as a series of independent chains of molecular weight $M^{*}$. Therefore, mixtures of small chains $M_{x}$ and long chains $M_{y}$ will be equivalent to the mixtures of chains $M_{x}$ and chains $M^{*}$ of the same weight concentration.

b) Mixture of high polydispersity. - The two mixtures $30 \mathrm{~K}-260 \mathrm{~K}$ and $10 \mathrm{~K}-260 \mathrm{~K}$ have polydispersities which are of the same order as that for commercial materials (between 4 and 10). As stated above, each long chain is equivalent to a series of smaller chains of mass $M^{*}$ (in our case two chains) therefore in the equivalent mixture $M_{x}, M^{*}$, the number and weight average of the end-to-end distance are given by the following equations :

$$
\begin{aligned}
& r_{\mathrm{w}}^{*}=\sum_{x} \omega_{x} M_{x}^{1 / 2}+\sum_{y} \omega_{y} M^{* 1 / 2} \\
& r_{\mathrm{N}}^{*}=\sum_{x} c_{x} M_{x}^{1 / 2}+\sum_{y} c_{y} M^{* 1 / 2}
\end{aligned}
$$

instead of equations (3) and (4).

In figure $6 a$ are shown the values of $L, r_{\mathrm{N}}, r_{\mathrm{w}}$ and $r_{\mathrm{w}}^{*}$ as a function of the number average molecular weight for the mixtures $10 \mathrm{~K}-260 \mathrm{~K}$.

From the figure, one concludes that the long period and the weight average of the end-to-end distance $r_{\mathrm{w}}$ present similar variations with molecular weight and that the long period is not correlated to the number average end-to-end distance $r_{\mathrm{N}}$.

The two parameters $L$ and $r_{\mathrm{w}}$ of the solid and liquid state level off for values of $M_{\mathrm{N}}$ above $M^{*}=10^{5}$. However, in this range of high molecular weight, the values of $L \sim 300 \AA$ and $r_{\mathrm{w}}=500 \AA$ (calculated from equation (3)) are quite different.

Considering the equivalent mixtures where each long chain is replaced by several smaller chains of mass $M^{*}$, it turns out that the weight average of the end-to-end distance $r_{\mathrm{w}}^{*}$ given by equation (5) follows the variations of the long periods and moreover these two parameters are of the same order of magnitude.

The correlation between $L$ and $r_{\mathrm{w}}^{*}$ is also observed for the mixture $30 \mathrm{~K}-260 \mathrm{~K}$ in figure $6 b$.

In conclusion, for mixtures of high polydispersity having long chains with $M>M^{*}$, the long period of the quenched semi-crystalline state is given by the relation (6) :

$$
L \sim r_{\mathrm{w}}^{*}
$$




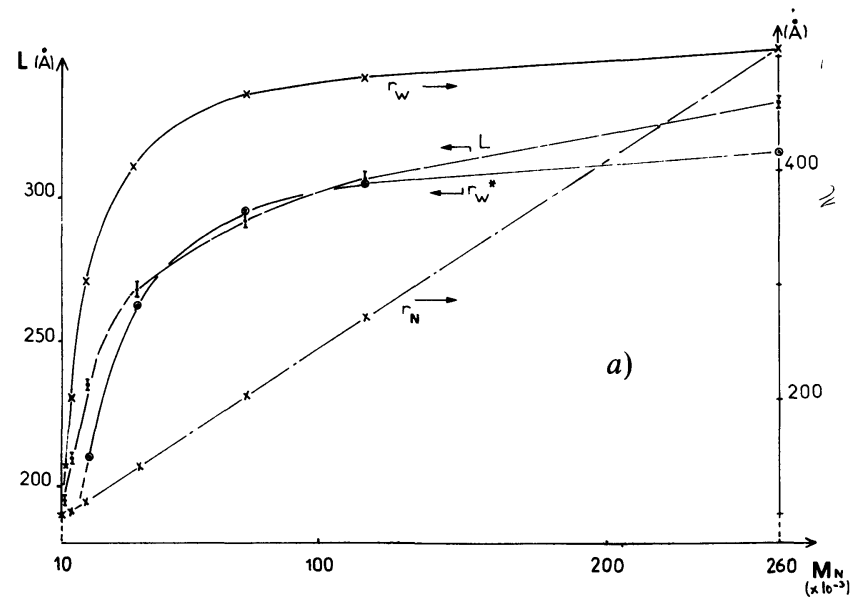

$\omega_{\mathbf{H}}$

$L$

1

$224 \AA$

0.5

$196 \AA$

0

$168 \AA$

The long period of the mixture of concentration $\omega_{\mathrm{H}}$ of PEH is $L \sim \omega_{\mathrm{H}} r_{\mathrm{H}}+\left(1-\omega_{\mathrm{H}}\right) r_{\mathrm{D}}$ that is to say, as $L_{\mathbf{H}} \sim r_{\mathrm{H}}$ and $L_{\mathrm{D}} \sim r_{\mathrm{D}}$ :

$$
L^{\bullet}=\omega_{\mathrm{H}} L_{\mathrm{H}}+\left(1-\omega_{\mathrm{H}}\right) L_{\mathrm{D}} .
$$

This result does not imply that the coefficient of proportionality between $L$ and $r$ is the same for PEH and PED. The mixture of concentration $50 \%$ will have, according to (7), a predicted long period of $196 \AA$. This is exactly the observed value.

We will show in a forthcoming paper that this linear law also applies for mixtures of high density and low density polyethylene.

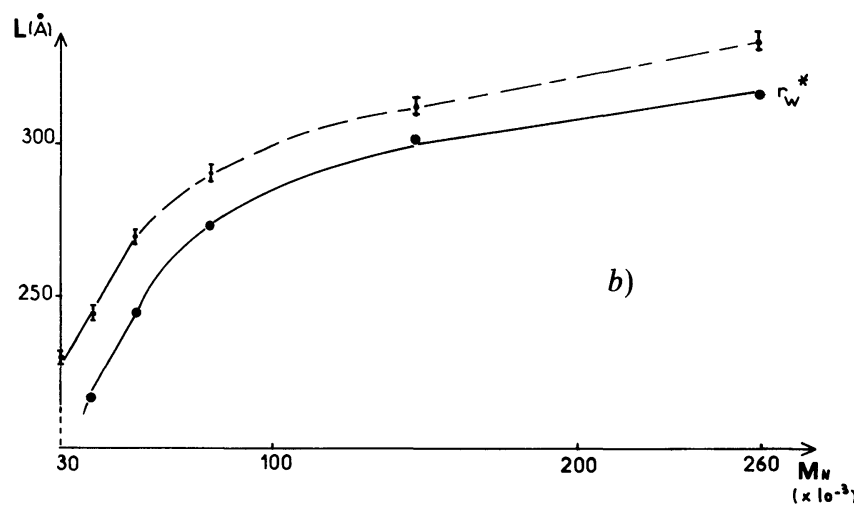

Fig. 6. - Correlations between the long period $L$ and the weight average $r_{\mathrm{w}}^{*}$ of the end-to-end distance of the coil in the liquid state before crystallization : a) mixture $10 \mathrm{~K}$ $260 \mathrm{~K} ; b$ ) mixture $30 \mathrm{~K}-260 \mathrm{~K}$.

where the weight average end-to-end distance of the equivalent mixture is given by (5).

From this relation, we obtain for monodispersed P.E. relations (1) and (2). Therefore, relation (6) is the general law of crystallization of monodispersed and polydispersed polyethylene. In a subsequent paper we will show that this law applies for a highly polydispersed P.E. having a unimodal distribution instead of a bimodal distribution as in our mixtures.

It is to be noted that this simple law (6) cannot be deduced from the various data in the literature because there has been no univocal relationship made between $L$ and the molecular weights $M_{\mathrm{w}}$ or $M_{\mathrm{N}}$, and systematic studies had not been undertaken.

c) Mixture of polydispersed polymers. - Let us consider two polydisperse polymers A and B of the same nature or of different natures which co-crystallize. In the quenched homopolymers the long periods are $L_{\mathrm{A}}$ and $L_{\mathrm{B}}$. In the quenched mixtures the long period will be given by the weight average of the end-to-end distances of the coils, according to relation (6).

The following table gives the values of $L$ of mixtures of hydrogenated and deuterated polyethylene-(PEHPED) as a function of the concentration $\omega_{\mathrm{H}}$ of PEH accordingly to Stamm et al. [11].
4.2 FLUCTUATION OF THE LONG PERIOD : INTERPRETATION OF THE WIDTH OF THE « BRAGG PEAK 》. Two models will be studied; the spinodal decomposition and the Tsvankin model, for deducing the dispersity $\Delta L / L$ of the long period from the width of the SAXS intensity peak.

a) Spinodal decomposition. - In oriented polymers some authors $[12,13]$ have suggested that the mechanism of crystallization is similar to the mechanism of phase separation in binary alloys [14], called spinodal decomposition. This comparison is based upon the two facts :

a) the intensity curve presents only one maximum (which is called improperly the Bragg peak);

b) the intensity curves at different times of annealing have a cross-over at a value $\theta_{\mathrm{c}}$ of the scattering angle. The intensity increases and decreases respectively below and above this value, with the time of annealing $[12,13]$.

Another important feature of the shear-induced crystallization of polymers has been given by Nogami et al. [52]. Their WAXS and light scattering studies lead to the conclusion that the process of shear induced crystallization involves the development of a lamellar organization and a mesomorphic state during transformation from the amorphous to the crystalline state. This observation is relevant with the spinodal decomposition mechanism and is incompatible with the secondary nucleation mechanism of crystallization.

The spinodal transformation occurs gradually and is characterized by density waves of wavelength $\Lambda$. There is no sharp frontier between crystal and amorphous phases. Each domain is instable. Domains with density waves $\Lambda>\Lambda_{\mathrm{c}}=\lambda / 2 \sin \theta_{\mathrm{c}} / 2$ decrease, and domains with $\Lambda<\Lambda_{\mathrm{c}}$ grow during the time of annealing. The transformation rate is maximized for $\Lambda_{\mathrm{m}}=\sqrt{2} \Lambda_{\mathrm{c}}$ according to Cahn [14]. The X-rays scattering presents a maximum for the corresponding scattering angle $\theta_{\mathrm{m}}=2 \arcsin 2 \Lambda_{\mathrm{m}} / \lambda$. 
The important point to note is that during the annealing process, during the earlier stages of the spinodal decomposition, the cross-over $\theta_{c}$ and the position $\theta_{\mathrm{m}}$ of the maximum of the scattering curve do not change. Therefore, the difference $\theta_{c}-\theta_{m}$ is constant. From the experimental results on binary alloys at various stages of annealing, it is found that the width of the intensity peak at half height is about $2\left(\theta_{c}-\theta_{m}\right)$. One concludes that the relative width of the intensity peak is :

$$
\frac{\Delta \theta}{\theta} \sim 2 \frac{\theta_{\mathrm{c}}-\theta_{\mathrm{m}}}{\theta_{\mathrm{m}}} \sim 0.8
$$

since $\theta_{\mathrm{c}} / \theta_{\mathrm{m}}=\sqrt{2}$.

In quenched fractions of high molecular weight $M>M^{*}$, the relative width is equal to 0.8 and independent of the molecular weight. This agreement with the observed value in binary alloys suggests strongly that the process of crystallization of quenched polymers and the spinodal decomposition of alloys are in some aspects very comparable.

As we have shown, the crystallization of long chains involves movement of only small segments of the full length $M^{*}$ of the chains. The spinodal decomposition implies a redistribution of different species, the two metals in binary alloys, defects and trans-trans bonds along the chains in the polymer melt.

In this model the long period, or the wave number $\Lambda_{\mathrm{m}}$ of the sinusoidal density fluctuation is given by :

$$
\Lambda_{\mathrm{m}}=c(1-c) \cdot \psi \cdot\left(\frac{T_{\mathrm{c}}}{\Delta T}\right)^{1 / 2}
$$

where $c$ is the concentration of defects,

$\psi$ an interaction distance,

$T_{\mathrm{c}}$ the temperature of crystallization,

$\Delta T$ the supercooling below the spinodal.

It is possible to fit this law in a small range of supercooling, with the well-known : equation resulting from the Lauritzen and Hoffman kinetic theory $[4,53]$

$$
L \sim \frac{K}{\Delta T}
$$

by adjusting the constants $c$ and $\psi$.

In a large domain of supercooling these two laws can be differentiated as a rule. However when the supercooling is large, relation (10) is no longer valid. It has been shown that the limiting value of the long period when $\Delta T$ increase is a characteristic of the polymer chains, and that the importance of the domain of temperature where relation (10) applies depends on the mobility of the chain in the solid state [29]. Chains with bulky side groups or forming hydrogen bonds verify relation (10) only in a very small domain of temperature of crystallization. P.E. chains are expected to have the highest mobility in the solid state, and therefore to verify relation (10) in a large domain. However, because of the rapid crystallization rate, it appears that the attainable domain of supercooling is small and therefore that the comparison between the observed temperature variations of the long period with those predicted by the spinodal decomposition theory, the Lauritzen and Hoffman theory [53] and the annealing theory [29] cannot be conclusive. We emphasize here, a major difficulty in understanding the crystallization process of polymers; several phenomenon can appear nearly simultaneously. The first stage of crystallization, supposed to be a process of spinodal decomposition can be followed by structural rearrangements due to annealing. This second structural rearrangement stage, as it has been shown for solution grown crystals [29], can lead to a long period given by relation (10).

In conclusion, the value of the width of the SAXS peak and the observed dependence of the long period on the supercooling are important features which are predicted correctly by the spinodal decomposition model. It is to be emphasized that the kinetic theories do not predict such a large value of the half width of the intensity peak of quenched materials.

However, to compare the two mechanisms of polymer crystallization and phase separation of alloys by spinodal decomposition, the kinetics of crystallization from the isotropic melt must be studied in great detail.

On the other hand, it should be noted that, for small chains, the spinodal decomposition cannot explain the variations of the long period and of the relative width of the SAXS intensity peak with the molecular weight. Finally we see no reason why the condition of constant kink concentration $c$ (or defects concentration) as required for a constant $L$ holds for small chains. These defects rejected from the crystalline phase would be evacuated from the chain because the chain does not connect two adjacent crystallites and chain ends are mostly localized in the amorphous phase.

Thus we suggest, it is very likely that the critical molecular weight $M^{*}$ separates two domains where two distinct process of crystallization are operative.

b) Tsvankin and Slutsker analysis. - The lamellar system is supposed to be a two phase system with transition zones. The distribution functions of the crystalline and amorphous layers having respectively a rectangular and an exponential form yield a disordered one-dimensional lattice. The scattering of X-rays at small angle by such a lattice has been calculated by several authors $[16,19]$. The intensity curves are functions of the parameters $\beta / \alpha=\Delta l_{\mathrm{c}} / l_{\mathrm{c}}$ and $\varepsilon=\delta / l_{\mathrm{c}}$ where $l_{\mathrm{c}}$ and $\Delta l_{\mathrm{c}}$ are the mean crystalline thickness and its fluctuation, and $\delta$ the transition zone thickness. The fit of these curves to the experimental intensity curve leads to values of the order of 0.2 for each of these two parameters.

The attractiveness of this particular model is that from the measurement of the Bragg long period $L$ and of the width of the intensity peak, it is possible to find the true long period $L_{\mathrm{t}}\left(L_{\mathrm{t}}=l_{\mathrm{a}}+l_{\mathrm{c}}\right)$ as well as the average thickness of both the amorphous and crystalline phases, $l_{\mathrm{a}}$ and $l_{\mathrm{c}}$. 
The calibration curves of Tsvankin [17] and Buchanan [18] relate the half width of the SAXS intensity curve $\Delta \theta / \theta$, the linear crystallinity $\chi_{1}$ and the parameter $x=L_{\mathrm{t}} / L$, to the parameter $2 x \cdot \Delta \theta / \theta$.

In figure 7 , the relative width $\Delta \theta / \theta$ has been plotted as a function of the crystallinity; curve 1 is deduced from the calibration curves of Tsvankin, $\Delta \theta / \theta$ and $\chi$ are the theoretical values, curve 2 relates the observed value of $\Delta \theta / \theta$ with the linear crystallinity $\chi_{1} \sim l_{\mathrm{c}} / L$ where $l_{c}$, the crystalline core thickness is supposed to be constant and equal to $150 \AA$, and curve 3 relates the observed value of $\Delta \theta / \theta$ with the experimental index of crystallinity measured by the WAXS technique, for the pure fractions.

The agreement between the theoretical and experimental curves 1 and 2, for $0.4<x<0.6$ indicates that this model of a 2-phase system with its distribution functions of the crystalline and amorphous layer thickness can be useful for determining the relative fluctuation $\Delta L / L$. A better agreement between these two curves is obtained if the value of $l_{\mathrm{c}}$ is $130 \AA$.

For a paracrystalline lattice of small dispersity of the long period, the relative width of the SAXS intensity peak is equal to $[24,25]$,

$$
\frac{\Delta \theta}{\theta}=\pi^{2}\left(\frac{\Delta L}{L}\right)^{2} .
$$

Vainshtein [25] has shown that this relation is only valid for dispersity $\Delta L / L$ smaller than 0.1 that corresponds to a half width $\Delta \theta / \theta \simeq 0.1$. Therefore, it is obvious that this relationship cannot be applied to semi-crystalline polymers for which the observed values of $\Delta \theta / \theta$ are between 0.2 and 1 . The lower values are obtained for solution grown crystals and for annealed samples.

Slutsker [2], following the Tsvankin analysis, has found a linear relationship between $\Delta L / L$ and $\Delta \theta / \theta$

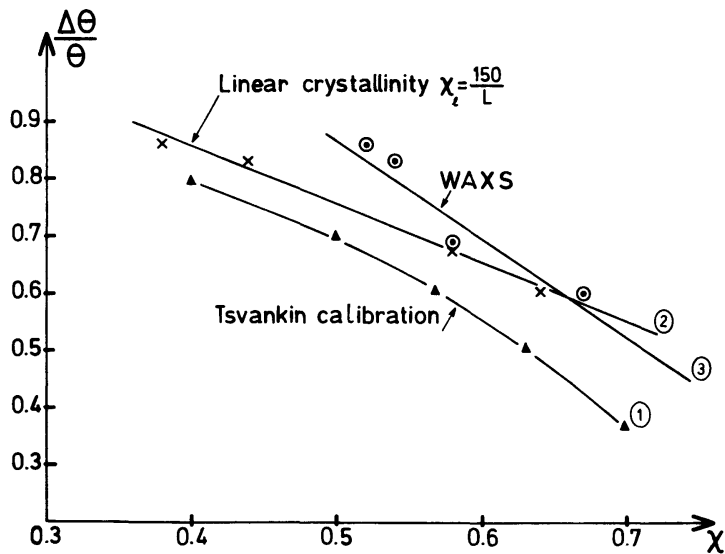

Fig. 7. - Curve 1 : calibration curve of Tsvankin, giving the relationship between the relative width of the Bragg peak $\Delta \theta / \theta$ at low angle and the crystallinity $\chi$. The experimental values of $\Delta \theta / \theta$ are expressed as a function of the predicted linear crystallinity $\chi_{1}=l_{\mathrm{c}} / L$ and as a function of the index of crystallinity measured by WAXS. for dispersity $\Delta L / L$ higher than 0.1 and lower than 0.5 . The theoretical curve is represented in figure 8 and, in the cases where the distribution of amorphous phase is Gaussian or statistical (Zernicke and Prins distribution) the relationship is the same for $0.1<\Delta \theta / \theta<0.5$ and differs somewhat above. This is represented in figure 8, curves. 1 and 2 . The mean curve between these two curves, curve 3 , is described by the relation :

$$
\frac{\Delta L}{L}=0.06+0.55 \frac{\Delta \theta}{\theta} .
$$

Slutsker and Kuzmin [21] have verified this relation by light scattering from models. The one-dimensional lattice with a relative fluctuation $\Delta L / L>0.1$ of the long period $L$ was imaged on a photographic plate and the incident light was such that :

$$
\frac{\lambda_{\text {light }}}{\lambda_{\text {R.X }}}=\frac{L_{\text {model }}}{L_{\text {polymer }}} .
$$

The results found using, the light scattering model are in good qualitative and quantitative agreement with the theoretical calculations of Slutsker. For values of $\Delta \theta / \theta$ from 0.1 to 0.5 relation (12) applies ; in this domain there is no difference in the theoretical curves if the type of distribution function of the long period is changed. For values of $\Delta \theta / \theta$ from 0.5 to 0.8 , the theoretical curves present some variations according to the type of distribution function of $L$. There is still a good agreement between the experimental curve of Slutsker and Kuzmin (see Fig. 5 of Ref. [21]) and the calculated curve assuming a Gaussian distribution of the long period. In this domain, the linear relationship between

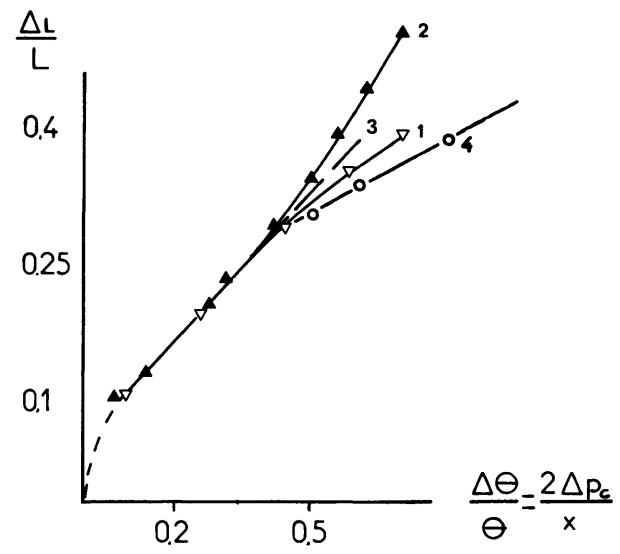

Fig. 8. - Dispersity of the long period $\Delta L / L$ expressed as a function of the half width $\Delta \theta / \theta$ of the SAXS intensity pattern : model of Tsvankin with a Gaussian distribution (1) and a distribution of Zernicke and Price (2). The mean curve (3) is given by relation (10). The experimental relationship curve 4 is deduced from small angle light scattering measurements on a fibrillar system : " light modelling method " of Slutsker and Kuzmin [21]. The dashed line is the curve predicted by the paracrystalline lattice theory in the case of weak dispersity $\Delta L / L<0.1$. 
the dispersity $\Delta L / L$ of the long period and the observed width of the SAXS and SALS pattern is :

$$
\frac{\Delta L}{L}=0.225+0.3 \frac{\Delta \theta}{\theta} .
$$

This experimental law deduced from the light scattering model is plotted in figure 8 , curve 4 .

For monodisperse P.E., the experimental values of $\Delta \theta / \theta$, by application of relation (13) lead to a constant dispersity of the long period :

$$
\frac{\Delta L}{L}=0.45
$$

independent of the molecular weight.

In conclusion, theoretical analysis by the Tsvankin and Slutsker models leads to a dispersity of the long period in quenched P.E. which is about 0.45 .

This conclusion obviously holds for mixtures of fractions and polydisperse P.E. because the relative width $\Delta \theta / \theta$ of these materials does not vary too much from those of the fractions.

The variations of $\Delta L / L$ for the mixtures studied in this work, as a function of $\sqrt{M}_{\mathrm{N}}$ or $\sqrt{M}_{\mathrm{w}}$ and deduced from relation (13) are very similar to those of the fraction.

Figure 4 shows the values of the dispersity $\Delta L / L$ for the fractionated samples. Curves 1 and 2 are obtained by application of relation (12) in which $\Delta \theta / \theta$ is respectively the experimental value (1) and the theoretical value (2) deduced from the Tsvankin calibration curve (deduced from the crystallinity, see Fig. 7). The intermediate dashed line 3 is obtained by application of relation (13), valid for large values of $\Delta \theta / \theta$.

Finally it is worth noting that the dispersity of about 0.5 , deduced from the Tsvankin and Slutsker analysis, is in agreement with the statistical evaluation of electron micrographs of low density P.E. crystallized at $100{ }^{\circ} \mathrm{C}$ (see Fig. 3 of Ref. [26]) which lead to a value of about 0.4. Several works have shown that the broadening of the SAXS peak is merely due to the dispersity of the long period, and not to the number of coherent scattering lamellae per macro-lattice [9]. Another broadening mechanism can be considered : the presence of domains of different long period (heterogeneous system); this would lead also to a linear relation [23] $\Delta \theta / \theta \simeq \Delta L / L$. However, the electron microscopy contradicts the existence of such domains in quenched materials $[26,27]$.

It is thus necessary to find a mechanism of crystallization which leads to a periodic lamellar system presenting a dispersity of the long period of the order of 0.5 independent of the molecular weight. We suggest in the following section that this value can be related to the dispersity of the end-to-end distance of the coils.

Remark on the homogeneity of the mixtures. - The segregation of chains of different mass $M_{x}$ and $M_{y}$ in different domains of size corresponding to the thick- ness of several lamella do not appear in our mixtures ; as a matter of fact the presence of two kinds of domains of long period $L_{x}$ and $L_{y}$ would lead to a SAXS intensity peak which should be resolved in two different Bragg peaks and this is not observed. The segregation, fractionation and clustering on a large scale of several long periods have not been observed in quenched polymers. In material crystallized slowly from the melt however, these phenomena appear on a large scale [11, 40-42]; an example is the segregation of different species (atactic chains, ...) at spherulite boundaries [4], and we think that segregation on a smaller scale of the order of $200 \AA$, also can appear; in other words, considering a random mixture of coils of different mass in the liquid state, it is not evident that these coils will be randomly distributed in the semi-crystalline state obtained by quenching the melt. As the smallest chains crystallize faster than the long chain, the centre of mass of these coils could have a non-uniform spatial distribution, in the direction perpendicular to the lamella.

In mixtures of small molecular weight fractions of P.E. $\left(M_{x}=2000, M_{y}=20000\right)$ it has been shown that segregation occurs during crystallization if the cooling rate is smaller than $20^{\circ} \mathrm{C} / \mathrm{min}$. [37].

4.3 Possible EXPLANATION OF THE DISPERSITY $\Delta L / L \sim 0.5$ OF THE LONG PERIOD. - As the long period is imposed by the end-to-end distance, according to the linear law $L \sim r$, it is possible that the fluctuation in the long periods results from the fluctuation of the end-to-end distances.

These correlations between chain conformation and chain statistics in the liquid and long period and dispersity of the long period in the semi-crystalline state can appear either at the beginning of the crystallization stage (during nucleation), or at the second stage during annealing. The exact mechanism of crystallization is out of the scope of this paper. However, one can suppose that when a nucleus, or a small part of an individual crystalline lamella is formed, the next adjacent nucleus has the greatest probability of being formed only at a distance of $L \sim r$. When the second nucleus is formed at that distance, the resulting adjacent lamella will grow with the same rate and stay at the same distance $L$ from the first lamella. The same process would apply for the nucleation of the second adjacent nucleus but with a different distance $L$ imposed by the different values of the dimension of the coil between the last crystalline layer and the new adjacent nucleus. This model suggests that chains cannot participate in two adjacent nuclei (or two adjacent crystalline lamellae). This can be easily understood by the following argument :

According to Roe et al. [44] and Krigbaum and Uematsu [45], the act of crystallization places tie chains if they do occur under high tension; we think that the increase in energy due to deformation of the amorphous tie chain, slows down the crystallization of the chain containing such a segment with respect to 
the other chains which do not participate in more than one crystalline nucleus; therefore, for that reason, nuclei containing no tie molecules will be more numerous than those which do. This is equivalent to saying that when a nucleus (or a lamellae is formed) the adjacent nucleus can nucleate at a distance from the centre of the first lamellae of the order of the end-to-end distance $r$ of the coils (in mixtures with $M_{\mathrm{i}}<M^{*}$ ). Nucleation at that distance $r$ will not lead to deformation of the amorphous chains. The observation that in mixtures and in polydisperse P.E. the long period is given by the number molecular weight rather than by the weight average molecular weight can be explained in this manner since the number of tie molecules increases with the difference $M_{\mathrm{w}}-M_{\mathrm{N}}$. For high molecular weight materials, $M_{\mathrm{N}}$ and $M_{\mathrm{w}}>M^{*}$, each chain must be considered as a tie molecule. It should be interesting to study the modulus of P.E. fractions and mixtures recognizing the influence of stressed tie molecules on that mechanical property.

In this model of crystallization the dispersity of the long period would be given by the dispersity of the end-to-end distance according to the law :

$$
\frac{\Delta L}{L}=\frac{\Delta r}{r} .
$$

The dispersity of the end-to-end distance is due to the Gaussian character of the chains and to the polydispersity of the length of the chains.

a) Effect of the Gaussian character of the coils. - Let us assume that the material is monodispersed, and of molecular weight $M<M^{*}$. The probability $w(r)$ of a coil having an end-to-end distance $r$ is [32] :

$$
w(r) \mathrm{d} r=\left(\frac{\beta}{\sqrt{\pi}}\right)^{3} \exp \left(-\beta^{2} r^{2}\right) .4 \pi r^{2} \mathrm{~d} r .
$$

where $\beta$ is related to the number $N$ of links of dimension $a$ of the chain by $\beta^{2}=3 / 2 N a^{2}$. The mean value and the quadratic mean value are :

and

$$
\bar{r}=\frac{2}{\beta \sqrt{\pi}}
$$

$$
\left(\overline{r^{2}}\right)^{1 / 2}=\frac{1}{\beta} \sqrt{\frac{3}{2}}=N a^{2}
$$

The dispersity of the distribution function is :

$$
\frac{\Delta r}{r}=\left[\frac{\overline{r^{2}}-\bar{r}^{2}}{\overline{r^{2}}}\right]^{1 / 2}=0.42 \text {. }
$$

This value, called hereafter the natural or Gaussian dispersity, is of the same order of magnitude as the experimental dispersity $\Delta L / L$ and likewise independent of the molecular weight. This correlation between the dispersity of the long period in the semicrystalline state and the natural dispersity of the end-to-end distance of the coils in molten monodispersed P.E., suggests that the law $L \sim r$ can be derived yielding relation (15) and that our simple model of crystallization is a possible model at least not contradicted by the experiments.

It must be recalled that at the present time no other explanation of the value of the dispersity $\Delta L / L$ in quenched polymers has been presented.

b) Effect of the polydispersity. - Let us assume that each coil has the end-to-end distance $r$, rather than the distribution of values given by equation (16). Let us assume that the distribution of the molecular weight is a arbitrary function, and that the number and weight averages of the molecular weight $M_{\mathrm{N}}$ and $M_{\mathrm{w}}$ are known. The width $\Delta M$ of this distribution function is then given by (32) :

$$
\overline{\Delta M^{2}}=M_{\mathrm{N}}\left(M_{\mathrm{w}}-M_{\mathrm{N}}\right)
$$

and the relative width is related to the polydispersity $I$ by :

$$
\frac{\Delta M}{M}=(I-1)^{1 / 2} .
$$

In that case, the law $L \sim \sqrt{M}$ leads to :

$$
\frac{\Delta L}{L}=\frac{1}{2} \frac{\Delta M}{M}=\frac{1}{2}(I-1)^{1 / 2} .
$$

This dispersity will be called non Gaussian dispersity as opposed to that in equation (17). For fractionated samples studied in this work, the polydispersity is of the order of 1.1. This leads to a dispersity $\Delta L / L=0.16$ considerably smaller than the natural dispersity, relation (17), due to the Gaussian character of the coil. This is the reason why the effect of polydispersity in fractionated P.E. is neglected for explaining the dispersity of the long period.

In the general case of mixtures of fractionated samples and of highly polydispersed samples, the probability $W(r) \mathrm{d} r$ of having a coil with end-to-end distance in the range $r$ to $r+\mathrm{d} r$ (proportional to the number concentration of coils having a end-to-end distance $r$ ) is given by relation (19):

$$
W(r) \mathrm{d} r=\int_{M} f(M) \cdot w(r) . \mathrm{d} r . \mathrm{d} M
$$

where $f(M)$ is the distribution function of the molecular weight and $w(r) \mathrm{d} r$ the distribution of the end-toend distance dependent on $M$ and given by equation (16). By equation (19), the dispersity $\Delta r / r$ can be calculated. This complicated task will not be done here because the effect of the molecular weight distribution is small in any case.

For high molecular weight mixtures $M>M^{*}$, the chains have been decomposed into smaller parts of molecular weight $M^{*}$ and of virtual end-to-end 
distance $r^{*}$. It is natural to assume that this distance has a fluctuation of the order of $\Delta r^{*} / r^{*} \sim 0.42$ as for Gaussian chains. With regard to crystallization, the mixture is equivalent to a fractionated sample of molecular weight $M^{*}$.

For mixtures of short chains, the dispersity $\Delta L / L$ due to the polydispersity $I$ is of the same order of magnitude as the dispersity due to the Gaussian character if $I \geqslant 1.71$. This value is not reached for our mixtures of $50 \%$ of $30 \mathrm{~K}-100 \mathrm{~K}$ and $30 \mathrm{~K}-60 \mathrm{~K}$. In those materials, the maximum of polydispersities are, respectively, 1.12 and 1.3 , thus permitting us to neglect the non Gaussian dispersity given by equation (18).

The mixtures of small and long chain, as we have pointed out, are equivalent to mixtures with long chains of mass $M^{*}$. The non Gaussian dispersity would be given by equation (18) in which the polydispersity $I$ is replaced by the polydispersity of the equivalent mixture.

For the mixture $10 \mathrm{~K}-260 \mathrm{~K}$, the polydispersity of the $50 \%$ mixture is 7 but that of the equivalent mixture $10 \mathrm{~K}-100 \mathrm{~K}$ is 3 . In that case the non Gaussian dispersity $\Delta L / L$ is about 0.7 and thus larger than the Gaussian dispersity. Only in these particular mixtures must the effect of the molecular weight polydispersity be taken into account. For that particular example the values of $M_{\mathrm{N}}$ and $M_{\mathrm{w}}$ are respectively 20000 and 134000 . The difference between these two values is somewhat greater than that of commercial polydisperse P.E.

In all the mixtures studied, the relative half width $\Delta \theta / \theta$ presents a maximum for a concentration of long chains of about 75 to $80 \%$ instead of $50 \%$ which would be expected if relation (18) applies. The reason for the difference between the maximum in $\Delta \theta / \theta$ (and $\Delta L / L$ ) and $I$ as a function of the concentration of the mixtures is puzzling. It is to be born in mind that the mechanism of crystallization which leads to the relation $L \sim r$ does not lead automatically to the conclusion that the fluctuation of the long period $\Delta L$ is related to the fluctuation $\Delta r$ of the end-to-end distance. What we point out is that these two fluctuations of the structural parameters $L$ and $r$ characteristic of the solid and liquid phase are of the same order of magnitude and vary in the same way with the molecular weight, and consequently they may be related, suggesting therefore that the mechanism of crystallization proposed here is not irrelevant.

However, in a two stage crystallization, rearrangement of the semi-crystalline structure can appear during the annealing that accompanies crystallization $[11,29]$. This process, without changing the long period, could change its fluctuation and probably several mechanisms of rearrangement can be imagined for explaining the value of the dispersity : the spinodal decomposition mechanism is one example shown in a former section.
5. Crystallinity of quenched P.E. - It is evident that the paramount question concerning the semicrystalline state of polymers is : what are the parameters governing the crystallinity of these materials? It is well-known that the crystallinity is a function of the supercooling $\Delta T[33,43,45]$ but at high supercooling $\Delta T \gg 20^{\circ} \mathrm{C}$, the crystallinity is a constant whose value is dependent on the molecular weight [43] and not on $\Delta T$.

The quenching of a sample in water or in liquid nitrogen leads to materials with the same crystallinity. This value of the crystallinity is a characteristic parameter of the solid phase depending on the nature of the polymer [33] in the same manner as the crystalline lamellae core thickness $l_{\mathrm{c}}$ [30] and the long period $L$ analysed in this paper and in Part II. In the model of crystallization proposed by one of the authors [29-31], the limiting value of $l_{\mathrm{c}}$ obtained at high supercooling, is about twenty persistence lengths for solution grown crystals and about twice that value for quenched polymers. That value is of the order of magnitude of the curvilinear distance between two consecutive entanglement points along one chain [31]. In both cases, $l_{c}$ is only a function of the rigidity of the chain, and independent of the molecular weight. That last property has not been verified by Raman scattering studies of the low frequency modes. However, in first approximation we will assume in this work that $l_{\mathrm{c}}$ is constant or more exactly that $l_{\mathrm{c}}$ does not vary as much as $L$ with the molecular weight. This is suggested by the following facts :

a) Raman scattering and indirect techniques on different P.E. materials with different molecular weight distributions $[36,54]$ give $l_{\mathrm{c}}$ values of about 150 to $170 \AA$.

b) The product $\chi . L$ for sharp fractions is constant and equal to $130 \AA$ [2].

c) The exact relationship between $M$ and $L$ for sharp fractions is $L=130+0.54 \sqrt{M}$ (see equation (4) in Ref. [2]). The constant term in that equation is of the order of magnitude of $\chi L$ and of the entanglement distance in the melt. The comparison of that equation with the relation $L=l_{\mathrm{c}}+l_{\mathrm{a}}$ suggests that $l_{\mathrm{c}} \sim 130 \AA$ and $l_{\mathrm{a}} \sim 0.54 \sqrt{M}$; because the crystallinity decreases with the molecular weight, the amorphous layer thickness $l_{\mathrm{a}}$ is expected to increase with $M$.

As noted by Capaccio et al. [46, 47], the value of $l_{\mathrm{c}}$ measured by X-rays is about $20 \%$ smaller that the value measured by low frequency Raman spectroscopy. We assume that the $l_{\mathrm{c}}$ value is about $150 \AA$. The expected linear crystallinity is then :

$$
\chi_{1}=\frac{150}{L_{\mathrm{c}}} .
$$

We have compared the linear and mass crystallinity in figures 7 and 9 . In figure 7 , the comparison 

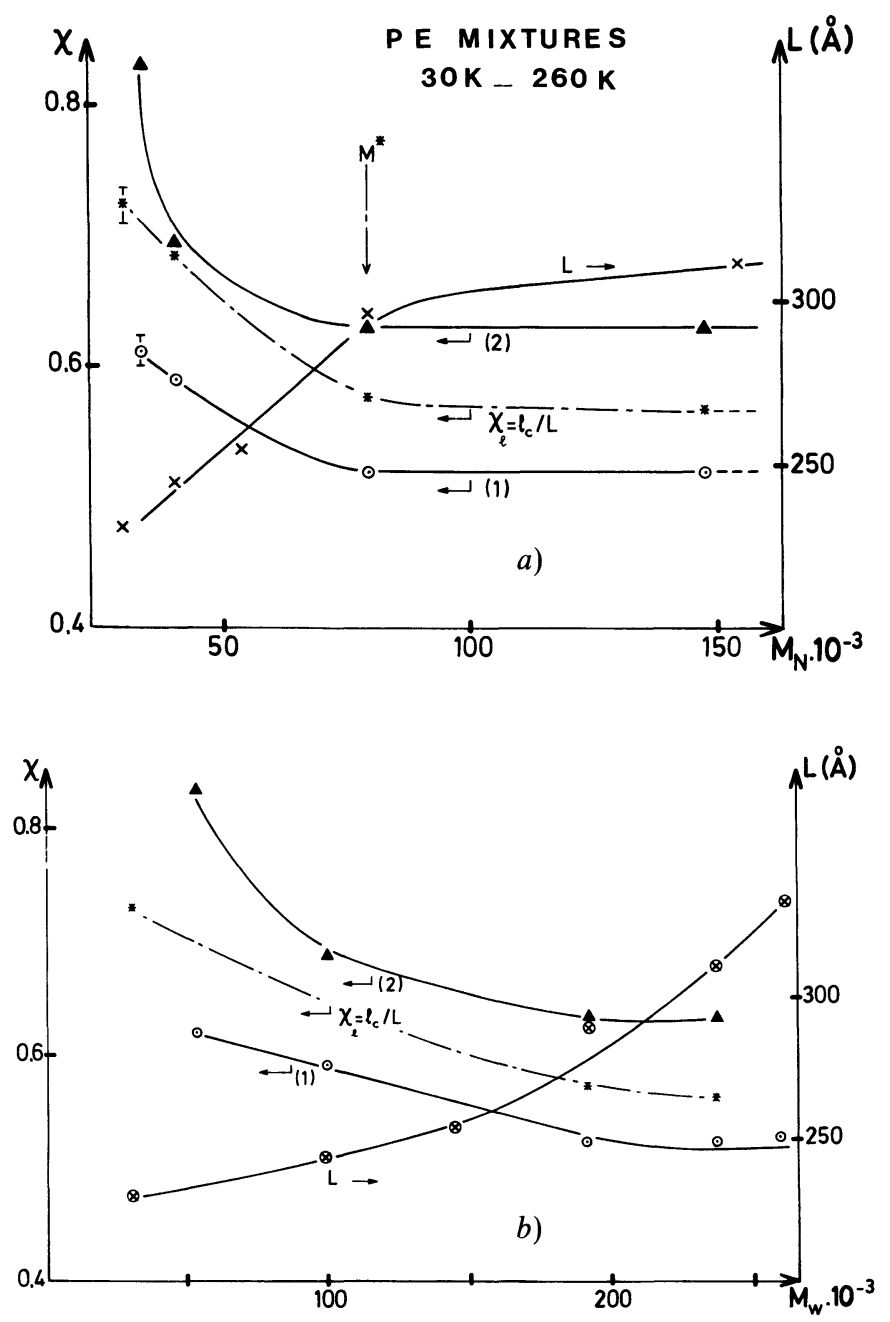

Fig. 9. - Crystallinity of mixtures $30 \mathrm{~K}-260 \mathrm{~K}$ expressed as a function of the number $(a)$ and weight average $(b)$ of the molecular weight. Curves 1 and 2 : DTA measurements for quenched (1) and slow cooled materials (2). Dashed line : the linear crystallinity $\chi_{1}$ deduced from the value of the long period, for quenched materials.

with the index of crystallinity measured by WAXS shows that the higher is $\Delta \theta / \theta$, (the higher is the molecular weight according to figure 1), the lower the crystallinity and the greater the deviation between the two indices of crystallinity. That means that the amount of non-interlamellar amorphous phase (inter spherulitic amorphous phase) increases with the molecular weight. This is obviously relevant to the observation that the perfection of the spherulitic structure decreases with the molecular weight $[28,35$, $48,51]$.

In figure 9, the values of expected linear crystallinity and those of the mass crystallinity measured by DTA are shown. Here again, it is to be noted that in quenched samples $\chi(\mathrm{X}$-ray $)<\chi($ linear $)$ and that these two parameters present similar variations with the two molecular weights $M_{\mathrm{N}}$ and $M_{\mathrm{w}}$. When $\chi$ is plotted as a function of $M_{\mathrm{N}}$, a transition is observed for $M_{\mathrm{N}} \sim 75000$. The same transition is observed for the long period. It is to be noted that the transition is very near the value of the critical mass $M^{*}$ observed for fractionated P.E. as shown in figures 3 and 4, and which separates the two domains of molecular weight.

It is to be noted that the similarity between the variations of the linear and the mass crystallinity does not depend too much on the quenching rate, whereas the absolute values are very dependent. This conclusion is drawn from the comparison of the two mass crystallinity curves, 1 and 2, obtained for quenched materials and for materials crystallized at a cooling rate of $2.5^{\circ} \mathrm{C} / \mathrm{min}$.

The same conclusions are reached for the other mixtures; figures 10 and 11 give the dependence of

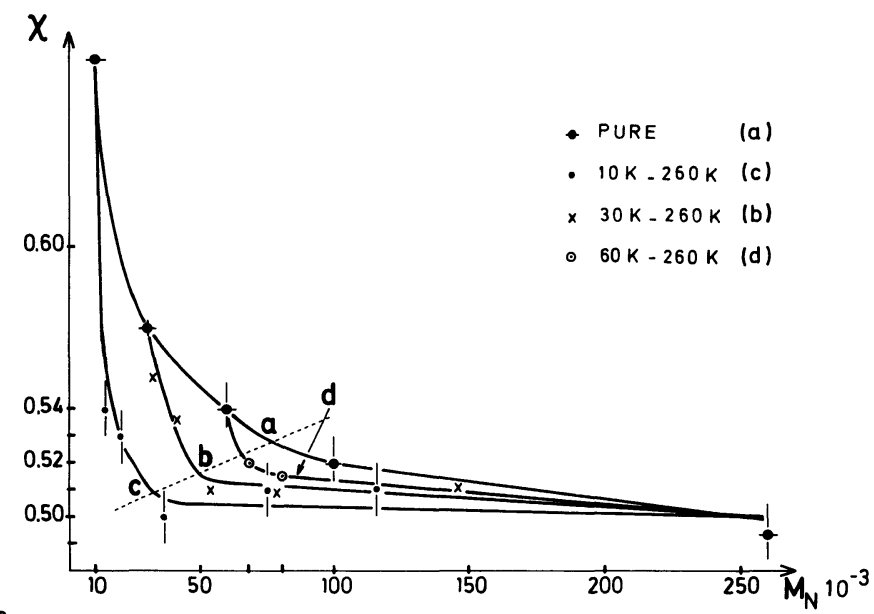

Fig. 10. - Index of crystallinity of P.E. mixtures deduced from WAXS measurements against the number molecular weight. For each type of mixture, a critical mass is defined.

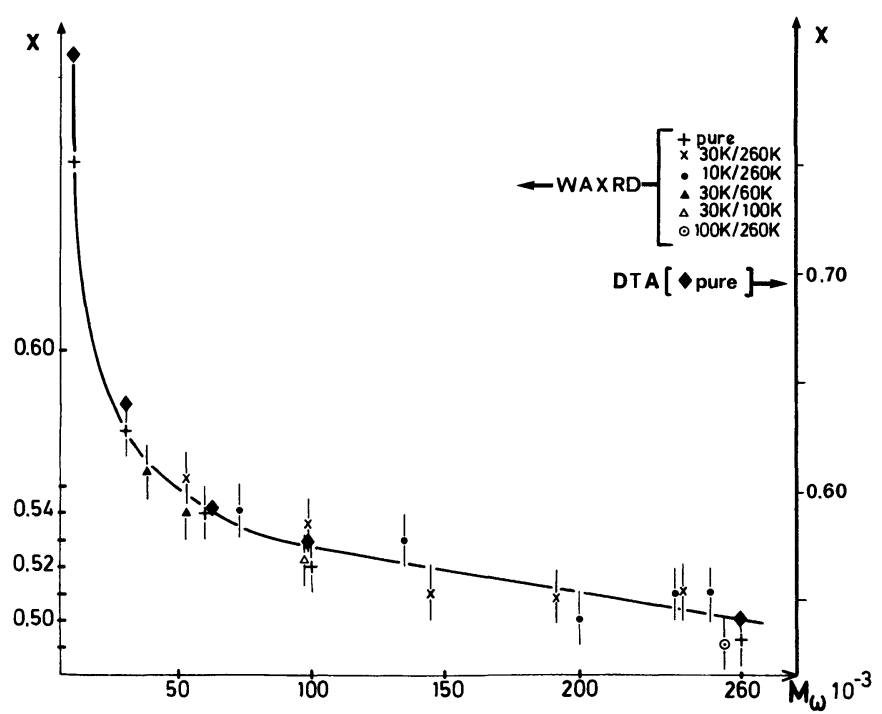

Fig. 11. - Index of crystallinity measured by WAXS analysis and by Differential Thermal Analysis (DTA) expressed as a function of the weight average of the molecular weight of the quenched mixtures. 
the WAXS crystallinity index on the molecular weight $M_{\mathrm{N}}$ and $M_{\mathrm{w}}$. It is noted again that $\chi($ DTA $)>\chi($ WAXS $)$. A master curve $\chi=f\left(M_{\mathrm{w}}\right)$ is defined. This finding shows that having a P.E. fraction or a mixture of fractions, it is possible as a rule to determine the weight average molecular weight by measuring the crystallinity. However the accuracy of the measurements and the dispersity of the measured values reported in figure 11 impedes the application of the method.

In figure 10 , is shown a feature which was not so well observed with regard to the long period : the critical molecular weight of the different mixtures, lying on the dashed line, is a linear function of the molecular weight of the smallest chains. This critical mass above which the crystallinity is constant, is found to correspond to a constant coil concentration of high molecular weight material of $10 \%$.

At the present time the interpretation of this feature cannot be given; it would be necessary to know the dependence of that critical mass or critical concentration with the molecular weight of the longest chains.

Figures $12 a$ and $b$ summarize, for the two highly polydispersed mixtures, the correlations between the liquid state (end-to-end distance and polydispersity) and the solid state (long period, dispersity of the long period and crystallinity). Two different domains of low and high molecular weight concentrations

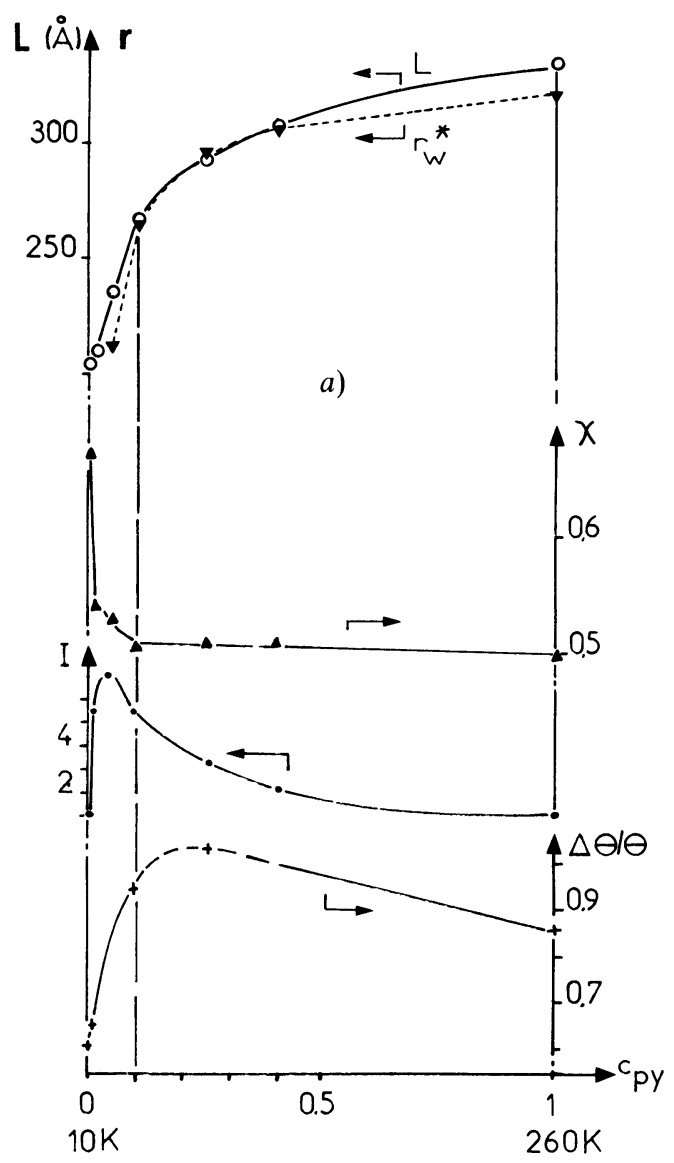

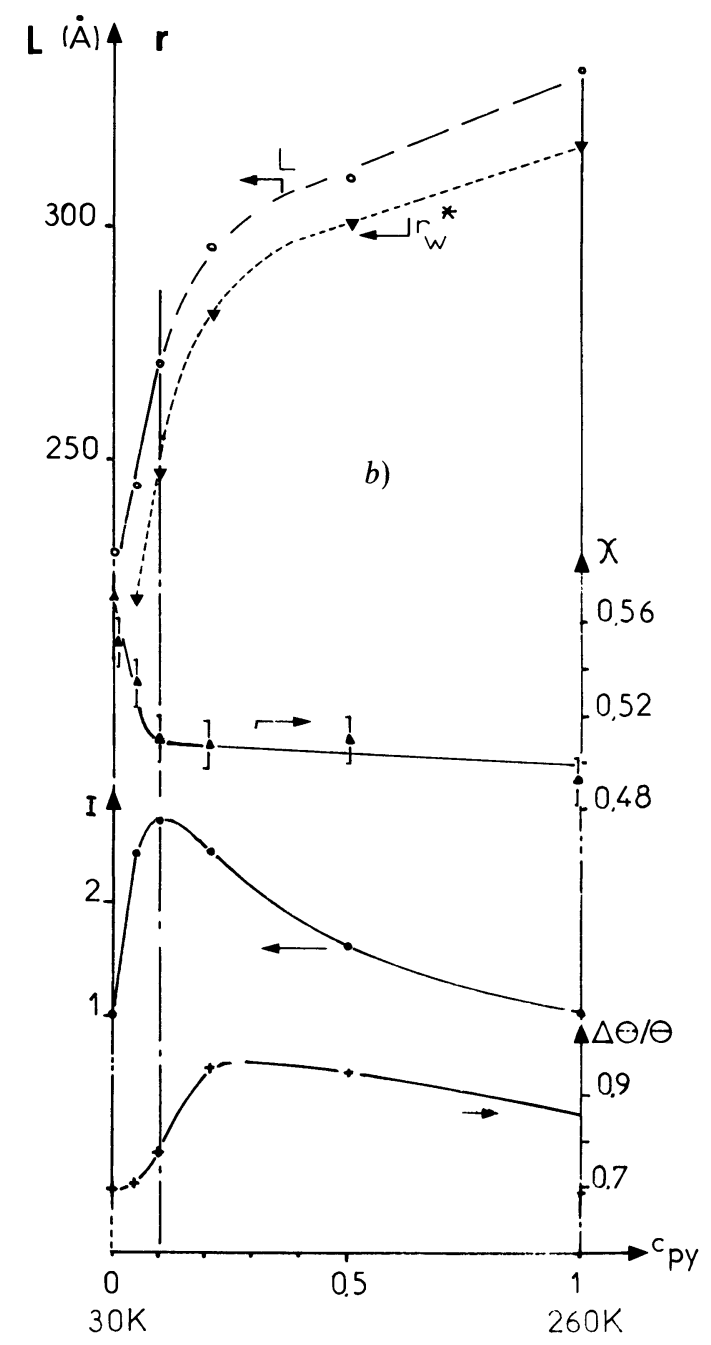

Fig. 12. - Correlations between the characteristics of the liquid chains (end-to-end distance $r_{\mathrm{w}}^{*}$ and polydispersity $I$ ) before crystallization and the characteristic of the semicrystalline state (long period $L$, crystallinity $\chi$ and the relative width $\Delta \theta / \theta$ of Bragg peak giving the dispersity $\Delta L / L)$. Quenched mixtures $10 \mathrm{~K}-260 \mathrm{~K}(a)$ and $30 \mathrm{~K}$ $260 \mathrm{~K}(b)$. The dashed line separates the two domains of crystallization of low and high molecular weight mixtures. Above the number coil concentration of $10 \%$ the characteristic parameters of the semi-crystalline state vary slowly with the concentration and with the molecular weight.

can be distinguished. The concentration of $10 \%$ separating these two domains corresponds to a weight average molecular weight of the mixture of the order of $M^{*}$, the critical mass observed in the study of sharp fractions.

6. Conclusion. - In mixtures of fractions of polyethylene, quenched from the melt, the correlations between the solid and liquid states enlighten the process of crystallization and support our interpretation of the influence of melt temperature on the long period of monodispersed and polydispersed 
polymers analysed in Parts I and II. The principal results of this work can be summarized as follow :

a) the long periods are given by the weight average of the end-to-end distance of the coils (Eqs. (3) and (5)) ;

b) expressed as a function of $M_{\mathrm{N}}$, the long periods vary as $\sqrt{M_{\mathrm{N}}}$ for $M_{\mathrm{N}}<M^{*}$ and are nearly constant above this critical mass;

c) the critical mass separating two domains of crystallization, corresponds to the critical mass $M^{*}=10^{5}$ found in the study of the crystallization of fractionated P.E. (Part II) and is of the order of magnitude of the molecular weight separating the domains of different supermolecular structure, and different crystallization rate $[28,48,49]$. This critical mass decreases somewhat when the molecular weight of the small chains in the mixture decreases, it corresponds to value of the molecular weight above which the crystallinity of quenched mixtures is constant at $\chi \simeq 0.5$;

d) the mass crystallinity and the linear crystallinity present the same variations with the molecular weight.

These features are simply explained by the law $L \sim r_{\mathrm{w}}^{*}$ where $r_{\mathrm{w}}^{*}$ is the weight average of the end-toend distances of the coils in the equivalent mixture before the quenching.

In this equivalent mixture the long chains of molecular weight greater than the critical mass have been replaced by chains of length $M^{*}$ which crystallize independently.

For small chains this law indicates that when a nucleus or a crystalline lamella is formed the next adjacent nucleus or crystalline lamella cannot be formed at a distance less than the end-to-end distance of the coils in the liquid state. This is explained by the fact that the crystallization of two adjacent nucleus or lamellae separated by a distance less than the unperturbated dimension $r$ of the coils would lead to a highly stressed amorphous phase. It is evident that at a distance greater than $r$ from a crystalline lamella, the movement of the chains are not hindered by the presence of that lamellae. Below that distance the movement of the chains are highly hindered. For these two reasons the end-to-end distance must be considered as a perturbation distance around a lamella, over which a new nucleus cannot nucleate, and a new crystallite cannot approach.

We have suggested that the polydispersity of the long period can be correlated to the dispersity $\Delta r / r$ of the coils in the liquid state. The application of the Tsvankin and Slutsker model of a two-phase system leads to a value of the polydispersity $\Delta L / L$ which is independent of the molecular weight and equal to the Gaussian dispersity. This value corresponds to that measured by electron microscopy [38]. The observed effect of the polydispersity on the value of $\Delta L / L$ is in general small compared to the effect of the Gaussian dispersity.

For long chains, a purely kinetic effect should explain the non-dependence of the long period on the molecular weight. We have shown that the process of spinodal decomposition which applies for segregation in alloys and for crystallization of stretched polymers can apply also to isotropic polyethylene of high molecular weight. This process of crystallization would explain the presence of only one SAXS intensity peak, and the width of the intensity peak, observed in quenched mixtures.

It is clear that in mixtures of fractions and in unimodal polydisperse P.E. containing short and long chains, there is not a unique mode of crystallization for all the chains. The relation (6) suggests that the two processes proposed in this paper apply also in polydisperse materials. That would explain the presence of different types of amorphous chains observed by N.M.R. and Raman scattering [35].

Finally, this work gives a clear definition of a tie molecule. In polydispersed P.E., every chain with a end-to-end distance greater than the weight average value $r_{\mathrm{w}}^{*}$ must be considered as a tie molecule. The number of tie molecules increases with the difference $M_{\mathrm{w}}-M_{\mathrm{N}}$. In general, in commercial material $M_{\mathrm{N}} \sim 20000$ whereas $M_{\mathrm{w}}$ varies from 50000 to $4 \times 10^{6}$. Hence, mechanical properties must be governed by the weight average molecular weight, this is observed in cold drawing studies [46, 47] and in fracture [50].

It has been shown that the properties of the bulk crystallized polymers are extremely dependent on the molecular weight [35]. In P.E. fractions [28, 49] and in mixtures of fractions [48] the supermolecular structures studied by small angle light scattering are also strongly dependent on the molecular weight. For quenched materials two types of spherulites are observed : type a for $M<5 \times 10^{5}$ and type $\mathrm{b}$ for $M>5 \times 10^{5}$. The molecular weight separating these two domains of superstructures is of the order of the critical molecular weight $M^{*}$ separating the two domains of crystallization of short and long chains studied in this work. The difference in the process of crystallization of short and long chains (difference in the scaling laws relations (1) and (2)) leads to a difference in the supermolecular structure.

In a subsequent paper, we will show that the correlations between the solid and liquid states found in P.E. exist also in the other polymers.

Acknowledgments. - We are grateful to Professor P. H. Geil for his remarks and criticisms about this work. 


\section{References}

[1] Rault, J., Sotton, M., Rabourdin, C., Robelin, E., J. Physique 41 (1980) 1459.

[2] Robelin, E., Rousseaux, F., Lemonnier, M., Rault, J., J. Physique 41 (1980) 1469.

[3] Rault, J., Robelin, E., Polym. Bull. 2 (1980) 373.

Robelin, E., Thesis 3e cycle, Orsay (1980).

[4] See for example GeIL, P. H., Polymer crystals (WileyInterscience N.Y.) 1963

Keller, A., Rep. Progr. Phys. 2 (1968) 923 ; Faraday Discuss. 69 (1979) 145.

[5] Sella, C., C. R. Hebd. Séan. Acad. Sci. Paris 248 (1959) 1819.

[6] KaKudo, M., KaSAI, N., X-ray diffraction of polymers (Elsevier Publ.) 1971.

Hermans, P. H., Weidinger, A., Makromol. 44 (1966) 24.

Statton, W. O., J. Appl. Polym. Sci. 7 (1963) 803.

[7] Magill, J. H., J. Polym. Sci. A 2 (1967) 89 ; Polymer 19 (1978) 416.

[8] Mandelkern, L., Acc. Chem. Res. 9 (1976) 81.

[9] CRIST, B., J. Polym. Sci. 11 (1973) 635.

Crist, B., Morosoff, N., J. Polym. Sci. 11 (1973) 1023.

[10] Haller, M., Magill, J. H., J. Appl. Phys. 40 (1969) 4261.

[11] Stamm, M., Fischer, E. W., Dettenmaier, M., ConVERT, P., Faraday Discuss. 68 (1979) 263.

[12] Schultz, J. M., J. Am. Phys. Soc. 25 (1980) 249.

Schultz, J. M., Lin, J. S., Hendricks, R. W., Peterman, J., Gohil, R. M., J. Mater. Sci.

[13] Fischer, E. W., GüNTher, Private Communication, January 1981.

[14] CaHn, J., Trans. Metal. Soc. 242 (1968) 167.

[15] Rundman, K. R., Hillard, J. E., Acta Metall. 15 (1967) 1025 .

[16] Hosemann, R., Bagchi, S. N., Direct analysis of diffraction by matter (North-Holland) 1962.

[17] Tsvankin, D., Vysokomol. Soedin. 6 (1964) 2304.

[18] Buchanan, D. R., J. Polym. Sci. 9 (1971) 645.

[19] Brämer, B., .Colloid. Polym. Sci. 252 (1974) 504.

[20] Slutsker, L. I., Polym. Sci. USSR 17 (1975) 303.

[21] Slutsker, L. I., Kuzmin, V. N., Vysokomol. Soyed A 17 (1975) 490.

[22] Strobl, G. R., Kolloid Z. Z. Polym. 250 (1972) 1039.

[23] Strobl, G. R., Müller, N., J. Polym. Sci. 11 (1973) 1219.

[24] Guinier, A., X-ray diffraction (Freeman and Company, San Francisco) 1963.

[25] Vainshtein, The X-ray diffraction by Molecular Chains (Izd. Akad. Nauk. URSS) 1963.

[26] Strobl, G. R., Schneider, M. J., Voigt-Martin, I. G., J. Polym. Sci. Phys. Ed. 18 (1980) 1361.
[27] Bassett, D. C., Hodge, A. M., Olley, R. H., Faraday Discuss. 68 (1979) 218.

[28] Voigt-Martin, I. G., Fischer, E. W., Mandelkern, L., J. Polym. Sci. Phys. 18 (1980) 2347.

[29] Rault, J., J. Physique-Lett. 39 (1978) L-411.

[30] Rault, J., J. Macromol. Sci. Phys. B 13 (1978) 4.

[31] Rault, J., Faraday Discuss. 68 (1979) 403.

[32] FloRY, P. J., Principle of Polymer Chemistry (Cornell University) 1953.

[33] Calvert, P., J. Polym. Sci. Phys. 17 (1979) 1341.

[34] Fatou, J., Mandelkekin, L., J. Phys. Chem. 69 (1965) 71.

[35] Mandelkern, L., a) Polym. Sci. Eng. 7 (1967) 232; b) J. Phys. Chem. 75 (1971) 3909; c) Faraday Discuss. 68 (1969) 310.

[36] Capaccio, G., Ward, I. M., Wilding, M. A., LongMan, G., J. Macromol. Sci. Phys. B 15 (1978) 381

[37] Smith, P., Manley, R. S. J., Macromolecules 12 (1979) 483.

[38] Strobl, G. R., Schneider, M., J. Polym. Sci. Polym. Phys. Ed. 19 (1980) 1343.

[39] Ruland, W., Colloid Polym. Sci. 5 (1977) 417.

[40] GeIL, P. H., Faraday Discuss. 69 (1979) 440.

[41] Ergoz, F., Fatou, J., Mandelkern, L., Macromolecules 5 (1972) 2, 147.

[42] Wunderlich, B., Faraday Discuss. 68 (1979) 239.

[43] Tung, L. H., Buckser, S., J. Phys. Chem. 62 (1958) 1530.

[44] Roe, R. J., Smith, J. R., Krigbaum, W. R., J. Chem. Phys. 5 (1964) 533.

[45] Krigbaum, W. R., Uematsu, I., J. Polym. Sci. A 3 (1965) 2915.

[46] Capaccio, G., Ward, I. M., Polymer 16(1975) 239.

[47] Capaccio, G., Crompton, T. A., Ward, I. M., J. Polym. Sci. Phys. 18 (1980) 301.

[48] Mandelkern, L., Go, S., Peiffer, D., Stein, R. S., J. Polym. Sci. Phys. 15 (1977) 1189.

[49] Maxfield, J., Mandelkern, L., Macromolecules 10 (1977) 1141.

[50] Rieumier, J. B., Thesis, Compiègne (1980).

[51] Fatou, J. G., Mandelkern, L., J. Phys. Chem. 69 (1965) 417.

Mandelkern, L., Allou Jr., A. L., Copalan, M., J. Phys. Chem. 72 (1968) 309.

[52] Nogami, K., Murakami, S., Katayama, K., Bull. Inst. Chem. Res. Kyoto 55 (1977) 227.

[53] Lauritzen, J. I., Hoffman, J. D., J. Res. Nat. Bur. Stand. A 64 (1960) 73.

[54] Hendra, P. J., Marsden, E. P., Polym. 15 (1977) 258.

[55] GeIl, P., J. Polym. Sci. C 13 (1966) 149. 